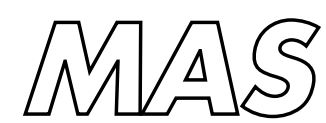

Modelling, Analysis and Simulation

Modelling, Analysis and Simulation
MAS An implicit-explicit Runge-Kutta-Chebyshev scheme for
diffusion-reaction equations

J.G. Verwer, B.P. Sommeijer

Report MAS-R0305 June 30, 2003 
CWI is the National Research Institute for Mathematics and Computer Science. It is sponsored by the Netherlands Organization for Scientific Research (NWO).

$\mathrm{CWI}$ is a founding member of ERCIM, the European Research Consortium for Informatics and Mathematics.

CWI's research has a theme-oriented structure and is grouped into four clusters. Listed below are the names of the clusters and in parentheses their acronyms.

Probability, Networks and Algorithms (PNA)

Software Engineering (SEN)

Modelling, Analysis and Simulation (MAS)

Information Systems (INS)

Copyright (C) 2003, Stichting Centrum voor Wiskunde en Informatica

P.O. Box 94079, 1090 GB Amsterdam (NL)

Kruislaan 413, 1098 SJ Amsterdam (NL)

Telephone +31205929333

Telefax +31205924199

ISSN 1386-3711 


\title{
An Implicit-Explicit Runge-Kutta-Chebyshev Scheme for Diffusion-Reaction Equations
}

\author{
J.G. Verwer and B.P. Sommeijer \\ $C W I$ \\ P.O. Box 94079, 1090 GB Amsterdam, The Netherlands \\ jan.verwer@cwi.nl (www.cwi.nl/ janv), ben.sommeijer@cwi.nl
}

\begin{abstract}
An implicit-explicit (IMEX) extension of the explicit Runge-Kutta-Chebyshev (RKC) scheme designed for parabolic PDEs is proposed for diffusion-reaction problems with severely stiff reaction terms. The IMEX scheme treats these reaction terms implicitly and diffusion terms explicitly. Within the setting of linear stability theory, the new IMEX scheme is unconditionally stable for reaction terms having a Jacobian matrix with a real spectrum. For diffusion terms the stability characteristics remain unchanged. A numerical comparison for a stiff, nonlinear radiation-diffusion problem between an RKC solver, an IMEX-RKC solver and the popular implicit BDF solver VODPK using the Krylov solver GMRES illustrates the excellent performance of the new scheme.
\end{abstract}

2000 Mathematics Subject Classification: Primary: 65M12, 65M20.

1998 ACM Computing Classification System: G.1.1, G.1.7 and G.1.8.

Keywords and Phrases: Diffusion-reaction equations, time integration.

Note: Work carried out under subtheme MAS1.3 - Numerical Analysis of PDEs

\section{Introduction}

This paper deals with the time integration of diffusion-reaction problems with highly stiff reaction terms. ${ }^{1}$ We adopt the method of lines approach and thus assume that the PDE problem including its boundary conditions has been spatially discretized to a semi-discrete problem

$$
w^{\prime}(t)=F_{D}(t, w(t))+F_{R}(t, w(t)), \quad t>0, \quad w(0)=w_{0},
$$

where $F_{D}$ represents the semi-discrete diffusion operator and $F_{R}$ contains the reaction terms. Typically, the dimension of this ODE system is huge, especially for multi-space dimensional PDEs and often this system is nonlinear and stiff. The stiffness rules out easy-to-use standard

\footnotetext{
1) A preprint of this work has been presented at the workshop Modelling and Simulation in Chemical Engineering, University of Coimbra, Portugal, June 30 - July 4, 2003.
} 
explicit solvers and the huge dimension with the nonlinearity complicates the use of implicit solvers.

We propose an implicit-explicit (IMEX) extension of the explicit Runge-Kutta-Chebyshev (RKC) scheme. This stabilized Runge-Kutta scheme for parabolic PDEs has been proposed by van der Houwen \& Sommeijer [7]. With respect to stability, it is positioned in between common explicit and implicit schemes. RKC is explicit and thus avoids algebraic system solutions. It does however possess an extended real stability interval with a length proportional to $s^{2}$, where $s$ is the number of stages. This quadratic dependence is derived from the first kind Chebyshev polynomial. The quadratic dependence implies that the scaled stability interval length, which takes into account the workload per time step (the number of stages), increases linearly with $s$. Therefore RKC is an attractive, user-friendly scheme for integrating large-scale semi-discrete parabolic problems. However, in case of severe stiffness, RKC will of course become inefficient since then a very large number of stages will be needed to achieve stability with reasonable step sizes.

The IMEX extension we propose is meant for problems (1.1) with a severely stiff reaction function $F_{R}$ and a moderately stiff diffusion function $F_{D}$. This extension treats $F_{D}$ still explicitly and $F_{R}$ implicitly. With a zero reaction term the original scheme is recovered, so that the IMEX extension maintains the attractive feature of the explicit scheme that no huge, coupled algebraic systems are to be solved. Only systems of small dimension (number of PDE components) coming from the reaction function are encountered. These small sized systems can be dealt with by the classic solution approach from stiff ODEs based on modified Newton iteration and standard LU-decomposition. Furthermore, the IMEX extension maintains the recursive Chebyshev nature such that we have stability for the testmodel

$$
w^{\prime}(t)=\lambda_{D} w(t)+\lambda_{R} w(t),
$$

for all real non-positive $\lambda_{D}$ and $\lambda_{R}$, as long as $\tau \lambda_{D}$ lies in the original real stability interval ( $\tau$ is here the step size). In this sense the IMEX scheme is unconditionally stable for the reaction part, assuming real eigenvalues.

In Section 2 we briefly review the explicit RKC scheme. The construction of the new IMEX scheme is discussed in Section 3. In Section 4 this new scheme is favourably compared with the popular BDF/GMRES solver VODPK [4, 6] for a highly stiff, nonlinear radiationdiffusion problem. For this purpose we have implemented the new IMEX scheme in the exisiting variable step size RKC solver from [15] which itself is also compared with the new IMEX solver. We conclude with the summarizing Section 5.

\section{The explicit RKC scheme}

Historically the principal goal when constructing Runge-Kutta schemes was to achieve the highest order possible with a given number of stages $s$. Stabilized methods are different in that only a few stages are used to achieve a usually low order whereas additional stages are exploited to increase the region of absolute stability, depending on the particular application. $\mathrm{RKC}$ is intended for semi-discrete parabolic PDE problems. Correspondingly, the scheme is stable on a strip containing a long segment of the negative real axis. The wider the strip, the greater the applicability of the method, but the most important characteristic of the formula is the length of the segment, the stability boundary $\beta(s)$. For ODEs resulting from semidiscretization, a low order formula is appropriate because usually only a modest accuracy is 
needed in solving PDEs. When the PDE involves more than one spatial variable, the size of the system of ODEs grows rapidly as the mesh spacing is decreased. The relatively crude meshes that are used for this reason lead to relatively large discretization errors in space, hence limits the accuracy that would be meaningful in the time integration and so favors low order methods. There exist RKC schemes of order 1 and 2 . Here we only consider the second-order scheme as this is believed to be more efficient than the first-order one.

The second-order scheme given in [14] is a slight improvement of the original secondorder scheme in [7]. A comprehensive linear stability and convergence analysis is found in [16]. Amongst others, this analysis proves that the RKC scheme does not suffer from order reduction. The schemes are also discussed in Ch.V of [8]. We refer to this text book for references to related work. A variable stepsize code based on the second-order scheme has been developed in $[15]^{2)}$ This code works with a variable number of stages to minimize computational costs. An IMEX version of this code will be used in Section 4.

Let $w_{n}$ denote the numerical approximation to the exact solution $w(t)$ of $w^{\prime}(t)=$ $F(t, w(t))$ at $t=t_{n}$ and let $\tau$ be the step size in the current step from $t_{n}$ to $t_{n+1}$. The second-order RKC scheme has the form

$$
\begin{aligned}
W_{0} & =w_{n}, \\
W_{1} & =W_{0}+\tilde{\mu}_{1} \tau F_{0}, \\
W_{j} & =\left(1-\mu_{j}-\nu_{j}\right) W_{0}+\mu_{j} W_{j-1}+\nu_{j} W_{j-2}+\tilde{\mu}_{j} \tau F_{j-1}+\tilde{\gamma}_{j} \tau F_{0}, \\
w_{n+1} & =W_{s},
\end{aligned}
$$

where $j=2, \ldots, s$ and $F_{k}$ denotes $F\left(t_{n}+c_{k} \tau, W_{k}\right)$. All coefficients are available in analytical form for arbitrary $s \geq 2$ :

$$
\begin{aligned}
& \tilde{\mu}_{1}=b_{1} \omega_{1} \text { and for } j=2, \ldots, s, \\
& \mu_{j}=\frac{2 b_{j} \omega_{0}}{b_{j-1}}, \quad \nu_{j}=\frac{-b_{j}}{b_{j-2}}, \quad \tilde{\mu}_{j}=\frac{2 b_{j} \omega_{1}}{b_{j-1}}, \quad \tilde{\gamma}_{j}=-a_{j-1} \tilde{\mu}_{j},
\end{aligned}
$$

for which the $a_{j}, b_{j}, c_{j}$ and $\omega_{0}, \omega_{1}$ are given below. Note the recursive form of $W_{j}$ by which only 5 arrays of storage are needed for all $s \geq 2$.

When applied to the scalar stability test equation $w^{\prime}(t)=\lambda w(t)$, we get at each stage a relation $W_{j}=P_{j}(z) W_{0}$ with $z=\tau \lambda$ and $P_{j}(z)$ a polynomial of degree $j$ in $z$ with $P_{s}(z)$ as stability polynomial. Formula (2.1) has in fact been derived from a particular set of functions $P_{j}(z)(0 \leq j \leq s)$ satisfying three design criteria: (i) nearly optimal step-by-step stability of $P_{s}(z)$ for parabolic problems, (ii) internal stability, i.e., controlled round-off accumulation in a single step for $s$ large, and (iii) second-order consistency of $P_{j}\left(c_{j} z\right)$ with respect to $e^{c_{j} z}$ for $j=2, \ldots, s$. Criterion (iii) automatically implies second-order consistency of all $W_{j}(2 \leq j \leq s)$ at $t=t_{n}+c_{j} \tau$ for general problems $w^{\prime}(t)=F(t, w(t))$. The first-stage formula is necessarily first-order consistent being forward Euler with step size $\tilde{\mu}_{1} \tau$.

The chosen functions $P_{j}$ are based on the first kind Chebyshev polynomials $T_{j}(x)$ satisfying the three-term recursion

$$
T_{j}(x)=2 x T_{j-1}(x)-T_{j-2}(x), \quad j=2,3, \ldots, s,
$$

where $T_{0}(x)=1, T_{1}(x)=x$. They are given by

$$
P_{j}(z)=a_{j}+b_{j} T_{j}\left(\omega_{0}+\omega_{1} z\right), \quad a_{j}=1-b_{j} T_{j}\left(\omega_{0}\right),
$$

2) See ftp://ftp.cwi.nl/pub/bsom/rkc or http://www.netlib.org/ode/ for the source code. 
where

$$
b_{0}=b_{1}=b_{2}, \quad b_{j}=T_{j}^{\prime \prime}\left(\omega_{0}\right) /\left(T_{j}^{\prime}\left(\omega_{0}\right)\right)^{2}, \quad j=2, \ldots, s,
$$

with $\omega_{0}=1+\epsilon / s^{2}, \omega_{1}=T_{s}^{\prime}\left(\omega_{0}\right) / T_{s}^{\prime \prime}\left(\omega_{0}\right)$ and containing $\epsilon \geq 0$ as a a free parameter. This parameter is called a damping parameter as it gives values of $P_{s}(z)$ strictly less than one in the interior of the real stability interval $[-\beta(s), 0]$.

Using $T_{s}^{\prime}(1)=s^{2}, T_{s}^{\prime \prime}(1)=\frac{1}{3} s^{2}\left(s^{2}-1\right)$ and $T_{s}^{\prime \prime \prime}(1)=\frac{1}{15} s^{2}\left(s^{2}-1\right)\left(s^{2}-4\right)$, the real stability boundary $\beta(s)$ can be seen to satisfy

$$
\beta(s) \approx \frac{\left(\omega_{0}+1\right) T_{s}^{\prime \prime}\left(\omega_{0}\right)}{T_{s}^{\prime}\left(\omega_{0}\right)} \approx \frac{2}{3}\left(s^{2}-1\right)\left(1-\frac{2}{15} \epsilon\right) .
$$

Taking $\epsilon=2 / 13$, we get approximately $0.33 \leq P_{s}(z) \leq 0.95$ in most of the interior of the stability interval and a reduction in the $\beta(s)$ of about $2 \%$ to $\beta(s) \approx 0.65\left(s^{2}-1\right)$ compared to the undamped case $(\epsilon=0)$. Figure 2.1 illustrates the stability region $\mathcal{S}=\left\{z \in \mathbb{C}:\left|P_{s}(z)\right| \leq\right.$ $1\}$ for $P_{5}(z)$ with and without damping. For larger values of $s$ similar regions exist, except more stretched to the left along the negative real line. Finally, the increment values $c_{j}$ are given by

$$
c_{0}=0, \quad c_{1}=\frac{c_{2}}{4 \omega_{0}}, \quad c_{j}=\frac{T_{s}^{\prime}\left(\omega_{0}\right)}{T_{s}^{\prime \prime}\left(\omega_{0}\right)} \frac{T_{j}^{\prime \prime}\left(\omega_{0}\right)}{T_{j}^{\prime}\left(\omega_{0}\right)} \approx \frac{j^{2}-1}{s^{2}-1} \quad(2 \leq j \leq s-1), \quad c_{s}=1 .
$$
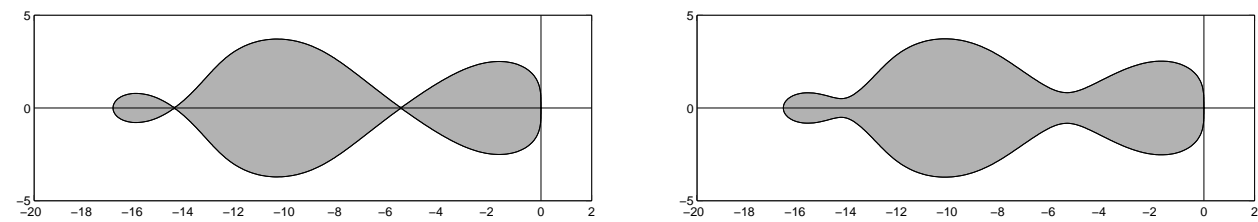

Figure 2.1: Stability regions for the second-order shifted Chebyshev polynomial $P_{5}$ : at the left the undamped case, at the right the damped case.

Remark 2.1 For $\epsilon=0$ we have

$$
P_{s}(z)=\frac{2}{3}+\frac{1}{3 s^{2}}+\left(\frac{1}{3}-\frac{1}{3 s^{2}}\right) T_{s}\left(1+\frac{3 z}{s^{2}-1}\right) \quad \text { with } \quad \beta \approx \frac{2}{3}\left(s^{2}-1\right) .
$$

This polynomial is due to [3] and generates about $80 \%$ of the optimal stability interval length for second-order polynomials, being $\beta(s) \approx 0.814 s^{2}$. Within most of the interior of the stability interval, $P_{s}(z)$ alternates between $\approx 1 / 3$ and 1 .

Remark 2.2 Related stabilized explicit methods are the ROCK $[1,2]$ and DUMKA methods $[9,10]$. These have close to optimal real stability boundaries and can possess a higher order (up to order 4). However, the formulas are not known in an explicit analytical form and are therefore less amenable for extension to an IMEX scheme. Numerical comparisons between the 2-nd order RKC code from [15] and a 4-th order ROCK code can be found in $[2,8]$. 


\section{The IMEX scheme}

\subsection{The integration formula}

We are now ready to construct the IMEX scheme for the general nonlinear system (1.1). For this system, the well-known IMEX-Euler scheme (see [8], Sect. 4.1) that is obtained from modifying the first stage formula of (2.1) reads

$$
W_{1}=W_{0}+\tilde{\mu}_{1} \tau F_{D}\left(t_{n}, W_{0}\right)+\tilde{\mu}_{1} \tau F_{R}\left(t_{n}+\tilde{\mu}_{1} \tau, W_{1}\right), \quad \tilde{\mu}_{1}=b_{1} \omega_{1} .
$$

Note that the reaction term is treated implicitly. All subsequent stages of (2.1) will be modified in a similar manner such that the recursive nature derived from the first kind Chebyshev polynomial is maintained.

Consider the scalar stability test equation (1.2) with $\lambda_{D}$ and $\lambda_{R}$ standing for eigenvalues of (frozen) Jacobians $F_{D}^{\prime}(t, w(t))$ and $F_{R}^{\prime}(t, w(t))$, respectively. Applied to this test equation, (3.1) yields

$$
W_{1}=R_{1}\left(z_{D}, z_{R}\right) W_{0}, \quad R_{1}\left(z_{D}, z_{R}\right)=\frac{1+b_{1} \omega_{1} z_{D}}{1-b_{1} \omega_{1} z_{R}} .
$$

As we will see, it is convenient to impose

$$
b_{1}=\frac{1}{\omega_{0}},
$$

so that

$$
R_{1}\left(z_{D}, z_{R}\right)=\frac{1+\frac{\omega_{1}}{\omega_{0}} z_{D}}{1-\frac{\omega_{1}}{\omega_{0}} z_{R}} .
$$

Observe that the choice (3.3) for $b_{1}$ differs from that made in (2.5). Here we exploit the freedom we have in choosing $b_{1}$ (like before, $b_{0}$ is again set equal to $b_{2}$ ). The current choice for $b_{1}$ enables the

Ansatz 3.1 All stage functions $R_{j}\left(z_{D}, z_{R}\right), j=0,1, \ldots, s$, of the IMEX scheme are taken to be of the form,

$$
R_{j}\left(z_{D}, z_{R}\right)=a_{j}+b_{j} T_{j}\left(\frac{\omega_{0}+\omega_{1} z_{D}}{1-\frac{\omega_{1}}{\omega_{0}} z_{R}}\right), \quad a_{j}=1-b_{j} T_{j}\left(\omega_{0}\right),
$$

with $b_{0}=b_{2}, b_{1}=1 / \omega_{0}$ and $b_{j}(j \geq 2)$ copied from $(2.5)$, so that for $z_{R}=0$ the $R_{j}$ reduce to the stage functions (2.4). Of importance is that the argument of the $T_{j}$ is identical over the stages.

The construction of the IMEX scheme is based on the rational function expression (3.5). First we write

$$
T_{j}(x)=\frac{-a_{j}}{b_{j}}+\frac{R_{j}}{b_{j}}, \quad x=\frac{\omega_{0}+\omega_{1} z_{D}}{1-\frac{\omega_{1}}{\omega_{0}} z_{R}},
$$

where $R_{j}=R_{j}\left(z_{D}, z_{R}\right)$ and apply the recursion (2.3). Inserting $x$ gives

$$
\begin{aligned}
& R_{j} \cdot\left(1-\frac{\omega_{1}}{\omega_{0}} z_{R}\right)=a_{j}\left(1-\frac{\omega_{1}}{\omega_{0}} z_{R}\right)+2 \frac{b_{j}}{b_{j-1}} R_{j-1} \cdot\left(\omega_{0}+\omega_{1} z_{D}\right)- \\
& 2 \frac{b_{j}}{b_{j-1}} a_{j-1}\left(\omega_{0}+\omega_{1} z_{D}\right)+\frac{b_{j}}{b_{j-2}} a_{j-2}\left(1-\frac{\omega_{1}}{\omega_{0}} z_{R}\right)-\frac{b_{j}}{b_{j-2}} R_{j-2} \cdot\left(1-\frac{\omega_{1}}{\omega_{0}} z_{R}\right) .
\end{aligned}
$$


From this relation we deduce the aimed IMEX integration scheme for system (1.1) by identifying the occurrence of $R_{j}$ with $W_{j}$ and of $R_{j} z_{R}$ with $\tau F_{R}\left(t_{n}+c_{j} \tau, W_{j}\right)$ and of $a_{j}$ with $a_{j} W_{0}$, etc. Using the coefficient expressions (2.2) this gives

$$
\begin{aligned}
& W_{j}-\tilde{\mu}_{1} \tau F_{R, j}=\left(a_{j}-\mu_{j} a_{j-1}-\nu_{j} a_{j-2}\right) W_{0}+\mu_{j} W_{j-1}+\nu_{j} W_{j-2}+ \\
& \tilde{\mu}_{j} \tau F_{D, j-1}+\tilde{\gamma}_{j} \tau F_{D, 0}-\nu_{j} \tilde{\mu}_{1} \tau F_{R, j-2}-\tilde{\mu}_{1}\left(a_{j}-\nu_{j} a_{j-2}\right) \tau F_{R, 0},
\end{aligned}
$$

where $F_{R, j}=F_{R}\left(t_{n}+c_{j} \tau, W_{j}\right)$, etc. Next, using $a_{j}-\mu_{j} a_{j-1}-\nu_{j} a_{j-2}=1-\nu_{j}-\mu_{j}$, we get

$$
\begin{aligned}
W_{0}= & w_{n}, \\
W_{1}= & W_{0}+\tilde{\mu}_{1} \tau F_{D, 0}+\tilde{\mu}_{1} \tau F_{R, 1}, \\
W_{j}= & \left(1-\mu_{j}-\nu_{j}\right) W_{0}+\mu_{j} W_{j-1}+\nu_{j} W_{j-2}+\tilde{\mu}_{j} \tau F_{D, j-1}+\tilde{\gamma}_{j} \tau F_{D, 0}+ \\
& {\left[\tilde{\gamma}_{j}-\left(1-\mu_{j}-\nu_{j}\right) \tilde{\mu}_{1}\right] \tau F_{R, 0}-\nu_{j} \tilde{\mu}_{1} \tau F_{R, j-2}+\tilde{\mu}_{1} \tau F_{R, j}, } \\
w_{n+1}= & W_{s},
\end{aligned}
$$

where $j=2, \ldots, s$.

Remark 3.2 If $F_{R}$ is absent, the explicit scheme (2.1) is recovered. For the diffusion operator $F_{D}$ the IMEX scheme thus operates in the same way as the explicit scheme. The difference is that (3.6) is implicit in the stiff reaction operator $F_{R}$, requiring at each stage the solution of a system of non-linear algebraic equations

$$
W_{j}-\tilde{\mu}_{1} \tau F_{R}\left(t_{n}+c_{j} \tau, W_{j}\right)=V_{j},
$$

with $V_{j}$ given and $W_{j}$ as unknown vector. Because $F_{R}$ has no underlying spatial grid connectivity, this system consists of a great number (the number of grid points) of decoupled small sized subsystems with dimension the number of coupled PDEs to be solved. Hence the modified Newton method can be used with a common LU-decomposition for the linear solves as is customary in the stiff ODE field. For efficiency reasons it could be advantageous that the coefficient $\tilde{\mu}_{1}$ is independent of $j$, since this would enable the use of LU-decompositions identical over the stages.

Remark 3.3 In many diffusion-reaction applications one is interested in transient behaviour and in steady-state solutions $w$ for autonomous problems

$$
F_{D}(w)+F_{R}(w)=0 .
$$

Standard Runge-Kutta and linear multistep methods return steady states exactly. This property is shared by all stages of the new scheme (3.6). It takes an elementary calculation to prove this. Note that with operator splitting where the subsystems $w^{\prime}(t)=F_{D}(w(t))$ and $w^{\prime}(t)=F_{R}(w(t))$ are integrated completely decoupled within time steps (time splitting), steady states are not returned exactly.

\subsection{Stability properties}

We consider (linear test model) stability for equation (1.2). The underlying assumption here, made for the sake of analysis, is that $\lambda_{D}$ and $\lambda_{R}$ stand for eigenvalues of frozen Jacobians 
$A_{D}=F_{D}^{\prime}(t, w(t))$ and $A_{R}=F_{R}^{\prime}(t, w(t))$, respectively, with $A_{D}$ and $A_{R}$ normal matrices which commute. They then have a common set of orthonormal eigenvectors, implying that stability results in the $L_{2}$-sense [8] for the constant coefficient linear system $w^{\prime}(t)=\left(A_{D}+\right.$ $\left.A_{R}\right) w(t)$ can be retrieved from the scalar equation $w^{\prime}(t)=\left(\lambda_{D}+\lambda_{R}\right) w(t)$. Additionally, we suppose that both $\lambda_{D}$ and $\lambda_{R}$ are real and non-positive; for many practical cases this imposes no restriction.

Thus, for the IMEX stability function

$$
R_{s}\left(z_{D}, z_{R}\right)=a_{s}+b_{s} T_{s}\left(\frac{\omega_{0}+\omega_{1} z_{D}}{1-\frac{\omega_{1}}{\omega_{0}} z_{R}}\right)
$$

we require $\left|R\left(z_{D}, z_{R}\right)\right| \leq 1$ for all possible values $\left(z_{D}, z_{R}\right)$ satisfying

$$
z_{D} \in[-\beta(s), 0] \text { and } z_{R} \leq 0 .
$$

Because $z_{R}$ is non-positive we have

$$
\left|\frac{\omega_{0}+\omega_{1} z_{D}}{1-\frac{\omega_{1}}{\omega_{0}} z_{R}}\right| \leq\left|\omega_{0}+\omega_{1} z_{D}\right|
$$

and it follows trivially that $\left|R_{s}\left(z_{D}, z_{R}\right)\right| \leq 1$ as long as $z_{D} \in[-\beta, 0]$, due to the fact that the coefficients $a_{s}, b_{s}$ were borrowed from the stability polynomial $P_{s}$ introduced in (2.4). Hence with respect to the reaction part the IMEX scheme is unconditionally stable and the stability with respect to the diffusion part remains unchanged.

For $z_{R} \rightarrow-\infty$ (infinite reaction stiffness) the argument of $T_{s}$ approaches zero. Hence for the IMEX scheme it is advocated to choose $s$ odd, giving $R_{s}\left(z_{D},-\infty\right) \approx 2 / 3$, or $s$ such that $T_{s}(0)=-1$, giving $R_{s}\left(z_{D},-\infty\right) \approx 1 / 3$. For both cases this would lead to a strong damping of stiff components from the reaction term.

\subsection{Consistency properties}

To see the change in consistency properties incurred by the IMEX extension, let us first examine how $R_{s}\left(z_{D}, z_{R}\right)$ approximates the exponential $e^{z}, z=z_{D}+z_{R}$, for $z \rightarrow 0$. For simplicity we put $\omega_{0}=1$ (no damping). Then the argument $x$ of $T_{s}$ in (3.8) satisfies

$$
x=\frac{1+\omega_{1} z_{D}}{1-\omega_{1} z_{R}}=1+\omega_{1} \tilde{z}, \quad \tilde{z}=\frac{z}{1-\omega_{1} z_{R}},
$$

with $\omega_{1}=3 /\left(s^{2}-1\right)$, so that we can write

$$
R_{s}\left(z_{D}, z_{R}\right)=\frac{2}{3}+\frac{1}{3 s^{2}}+\left(\frac{1}{3}-\frac{1}{3 s^{2}}\right) T_{s}\left(1+\frac{3}{s^{2}-1} \tilde{z}\right) .
$$

With $s$ sufficiently large and $\tilde{z} \rightarrow 0$ there holds [8]

$$
R_{s}\left(z_{D}, z_{R}\right) \approx 1+\tilde{z}+\frac{1}{2} \tilde{z}^{2}+\frac{1}{10} \tilde{z}^{3}+\cdots,
$$

giving

$$
e^{z}-R_{s}\left(z_{D}, z_{R}\right) \approx \frac{1}{15} z^{3}-\frac{3}{s^{2}-1} z_{R} z+\cdots
$$


We face a reduction of the order from two to one due to the IMEX extension. However, the new leading order term $3 z_{R} z /\left(s^{2}-1\right)$ vanishes with increasing number of stages, indicating that in actual application the effect of the order reduction will remain small.

Expansion (3.9) enables us to quickly assess the leading local error term for the nonlinear system (1.1). Let $w_{n}=w\left(t_{n}\right), w(t)$ being a sufficiently smooth exact solution. With a zero reaction term the explicit method then satisfies, by construction,

$$
\begin{aligned}
& W_{1}=w\left(t_{n}\right)+c_{1} \tau w^{\prime}\left(t_{n}\right), \\
& W_{j}=w\left(t_{n}\right)+c_{j} \tau w^{\prime}\left(t_{n}\right)+\frac{1}{2}\left(c_{j} \tau\right)^{2} w^{\prime \prime}\left(t_{n}\right)+\mathcal{O}\left(\tau^{3}\right), \quad j=2, \ldots, s .
\end{aligned}
$$

With a non-zero reaction term the order is restricted to one. Inspection of (3.6) then reveals that with a non-zero reaction term these expansions become

$$
\begin{aligned}
& W_{1}=w\left(t_{n}\right)+c_{1} \tau w^{\prime}\left(t_{n}\right)+\left(c_{1} \tau\right)^{2} S_{n}+\mathcal{O}\left(\tau^{3}\right) \\
& W_{j}=w\left(t_{n}\right)+c_{j} \tau w^{\prime}\left(t_{n}\right)+\frac{1}{2}\left(c_{j} \tau\right)^{2} w^{\prime \prime}\left(t_{n}\right)+\eta_{j} \tau^{2} S_{n}+\mathcal{O}\left(\tau^{3}\right), \quad j=2, \ldots, s
\end{aligned}
$$

and in particular

$$
w_{n+1}=w\left(t_{n}\right)+\tau w^{\prime}\left(t_{n}\right)+\frac{1}{2} \tau^{2} w^{\prime \prime}\left(t_{n}\right)+\eta_{s} \tau^{2} S_{n}+\mathcal{O}\left(\tau^{3}\right) .
$$

In line with $(3.9), S_{n}$ can be seen to be given by $S_{n}=F_{R}^{\prime}\left(w\left(t_{n}\right)\right) F\left(w\left(t_{n}\right)\right)$ where $F_{R}^{\prime}$ denotes the Jacobian of $F_{R}$ (we assume here the autonomous ODE form). The coefficient $\eta_{s}$ is defined by a complicated recursion formula. However, from (3.9) follows that for $s$ large $\eta_{s} \approx 3 /\left(s^{2}-1\right)$.

We have followed here the classical ODE error analysis. A more precise PDE error analysis, confirming second order for linear problems with a zero reaction term uniformly in the stiffness, has been given in [16]. This analysis extends to linear problems with a non-zero reaction term; this however is outside the scope of the current paper.

\section{Numerical illustration}

\subsection{A radiation-diffusion problem}

We will illustrate the new IMEX scheme for a radiation-diffusion problem from [11]. The following description and the used spatial discretization were borrowed from Ch.V of [8]. The problem consists of two strongly nonlinear diffusion equations with a highly stiff reaction term (an idealization of non-equilibrium radiation diffusion in a material). The dependent variables are $E$ and $T$, representing, respectively, radiation energy and material temperature. Problems like this are for instance found in laser fusion applications. The equations are defined on the unit square for $t>0$,

$$
E_{t}=\nabla \cdot\left(D_{1} \nabla E\right)+\sigma\left(T^{4}-E\right), \quad T_{t}=\nabla \cdot\left(D_{2} \nabla T\right)-\sigma\left(T^{4}-E\right),
$$

where $\sigma=Z^{3} / T^{3}, D_{1}=1 /(3 \sigma+|\nabla E| / E)$ and $D_{2}=k T^{5 / 2}$ with $k=0.005$. Further, $|\nabla E|$ is the Euclidean norm of $\nabla E$ and $Z=Z(x, y)$ represents the atomic mass number which may vary in the spatial domain to reflect inhomogeneities in the material. We have $Z(x, y)=Z_{0}$ if $|x-1 / 2| \leq 1 / 6$ and $|y-1 / 2| \leq 1 / 6$ with $Z_{0} \geq 1$ a constant and $Z(x, y)=1$ otherwise. 
The initial values are constant, $E(x, y, 0)=10^{-5}$ and $T(x, y, 0)=E(x, y, 0)^{1 / 4} \approx$ $5.6210^{-2}$. For boundary conditions we have homogeneous Neumann conditions for $T$ at all boundaries and for $E$ at $y=0,1$. Further, at the left and right boundary there holds $\frac{1}{4} E-\frac{1}{6 \sigma} E_{x}=1$ at $x=0$ and $\frac{1}{4} E+\frac{1}{6 \sigma} E_{x}=0$ at $x=1$.
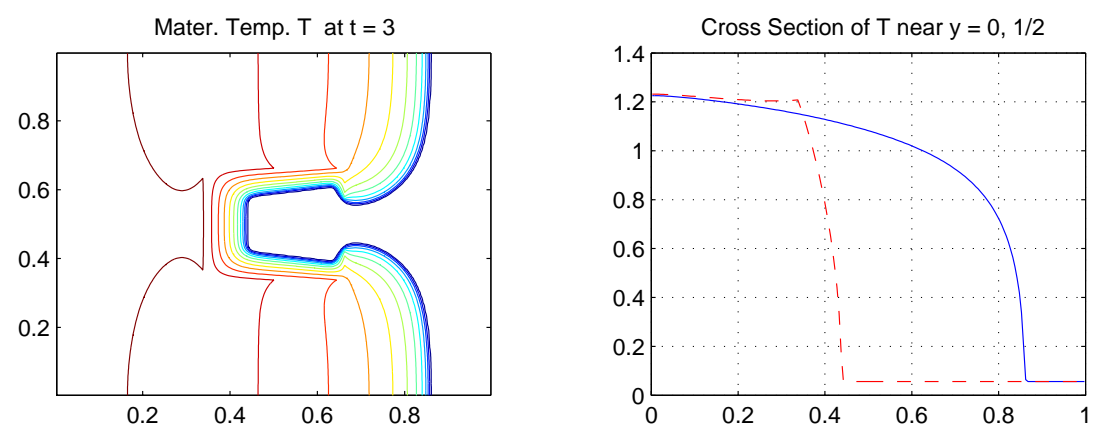

Figure 4.1: Contour levels and cross sections of the material temperature $T$ at time $t=3$ for $Z_{0}=10$. Contour levels: $0.1,0.2, \ldots, 1.2$.

The solution consists of a steep (temperature) front moving to the right. For $Z_{0}>1$ the movement is hampered at the interior region with larger atomic mass number (and corresponding smaller diffusion). $E$ is for the most part almost equal to $T^{4}$, except near the front where it is slightly larger with a steeper profile. Figure 4.1 shows contour levels and cross sections of an accurate reference solution of $T$ at $t=3$ for $Z_{0}=10$. More illustrations for different values of the temperature diffusion coefficient $(k=0,0.1)$ can be found in [11].

\subsection{Spatial discretization}

The spatial discretization is on a uniform cell centered grid with grid size $h$ by means of second-order central conservative differencing. This gives a semi-discrete system $w^{\prime}(t)=$ $F_{D}(w(t))+F_{R}(w(t))$ of dimension $2 / h^{2}$, for which the spectral radii of the frozen Jacobians $F_{D}^{\prime}, F_{R}^{\prime}$ are estimated as, respectively, $\rho_{D}=8 h^{-2}$ and $\rho_{R}=6000 Z_{0}^{3}$. Note that we have at each grid point the nonlinear reaction system

$$
f_{R}(E, T)=\left(\begin{array}{c}
Z^{3} T^{-3}\left(T^{4}-E\right) \\
-Z^{3} T^{-3}\left(T^{4}-E\right)
\end{array}\right), \quad f_{R}^{\prime}(E, T)=\left(\begin{array}{cc}
-\alpha & \beta \\
\alpha & -\beta
\end{array}\right),
$$

with $\alpha=Z^{3} / T^{3}, \beta=Z^{3}\left(1+3 E / T^{4}\right)$ and eigenvalues 0 and $-(\alpha+\beta)$. In the expression for $\alpha+\beta$ the term $Z^{3} / T^{3}$ will be the dominating one. Since we a priori know that $1 / T^{3} \lesssim 5.610^{3}$, we can estimate $\rho_{R}$ as $6000 Z_{0}^{3}$. In total we have as spectral radius $\rho_{D}+\rho_{R}$ which is to be maximized over the spatial region. With increasing atomic mass number $Z_{0}, \rho_{R}$ can quickly become much larger than $\rho_{D}$ for realistic grid sizes $h$. This is the kind of situation where the IMEX scheme will be significantly more efficient than its explicit counterpart. Recall that for the IMEX scheme we have the stability condition $\tau \rho_{D} \leq \beta(s)$, and for the explicit scheme $\tau\left(\rho_{D}+\rho_{R}\right) \leq \beta(s)$.

For the numerical comparison we have chosen $Z_{0}=10$ [11], giving $\rho_{R}=6.010^{6}$, and three realistic grid sizes, viz. $h=1 / 50,1 / 100,1 / 200$. This results in the spectral radii $\rho_{D}=2.010^{4}, 8.010^{4}, 3.210^{5}$, respectively. Especially on the two finer grids the diffusion 
part is rather stiff. Of course, if $\rho_{D}$ approaches $\rho_{R}$ the advantage of unconditional stability of the IMEX scheme for the reaction part decreases in comparison with the explicit scheme. Also observe that the finer the grid the better the front will be resolved. This will steepen up the temporal solution requiring a greater time integration effort (more time steps for a given temporal tolerance). The solution shown in Figure 4.1 has been computed on the $200 \times 200$ grid.

\subsection{Numerical results}

\subsubsection{Three solvers}

Numerical results are presented for

RKC: The solver from [15] based on the second-order explicit scheme (2.1). RKC works as most other variable step size ODE solvers. A difference is that at each time step it minimizes the number of stages $s$ so as to satisfy the stability condition $\tau \rho \leq \beta(s) \approx 0.65 s^{2}$, here with the prescribed $\rho=\rho_{D}+\rho_{R}$. Variable stepsizes are based on a local error per step criterion (which implies that if all is going well, the global error is reduced by a factor of roughly 5 upon a tolerance reduction factor of 10 [13]). A tentative initial step size $\tau_{0}=1 / \rho$ is used that is on scale with the dynamics at $t=0$. RKC requires 5 arrays of storage.

IMEX: This solver is based on (3.6) and is nearly identical to the solver RKC. It uses the same control strategies ${ }^{3}$ ) and differs only in the used spectral radius $\rho=\rho_{D}$ and in the additional solution of the reaction systems (3.7). Standard modified Newton iteration is used with a Jacobian update and LU-decomposition per stage and grid point. Acceptance for the iterants is thus decided per grid point, allowing the number of iterations to differ over the grid. As start vector the accepted iterant of the previous stage is used and the iteration is terminated as soon as the modified Newton correction is smaller than $1 \%$ of the imposed local error tolerance Tol, or as soon as the residual is less than $10^{-9}$ (both measured in the maximum norm). For the radiation-diffusion problem the modified Newton computations consume altogether about $40 \%$ to $50 \%$ of the total CPU time, using an average of about 1.3 iterations. IMEX requires 9 arrays of storage.

VODPK: The stiff solver VODE [4, 6] provided with the Krylov solver GMRES [12] with user-supplied preconditioner for solving the linear systems arising in the modified Newton iteration. ${ }^{4}$ In [5] a parallel version is used for solving a related problem (a single PDE coupled to a single ODE). For the radiation-diffusion problem preconditioning is essential. Without preconditioning VODPK either fails or is very inefficient, depending on the tolerance and the grid size. We have implemented a $2 \times 2$ block-diagonal left preconditioner $P$ which approximates the $2 \times 2$ block-diagonal of the Newton matrix. $P$ is derived from the grid point formula

$$
E_{i j}^{\prime}=-\frac{4}{h^{2}} D_{1, i j} E_{i j}+\sigma_{i j}\left(T_{i j}^{4}-E_{i j}\right), \quad T_{i j}^{\prime}=-\frac{4}{h^{2}} D_{2, i j} T_{i j}-\sigma_{i j}\left(T_{i j}^{4}-E_{i j}\right),
$$

where $D_{1}$ is approximated by $1 / 3 \sigma$. So the $P_{i j}$-block for grid point $\left(x_{i}, y_{j}\right)$ reads

$$
P_{i j}=\left(\begin{array}{cc}
1 & 0 \\
0 & 1
\end{array}\right)+b_{0} \tau \frac{4}{h^{2}}\left(\begin{array}{cc}
\frac{1}{3 \sigma_{i j}} & \frac{T_{i j}^{2} E_{i j}}{Z_{i j}^{3}} \\
0 & \frac{7}{2} k T_{i j}^{5 / 2}
\end{array}\right)-b_{0} \tau\left(\begin{array}{cc}
-\sigma_{i j} & Z_{i j}^{3}\left(1+3 \frac{E_{i j}}{T_{i j}^{4}}\right) \\
+\sigma_{i j} & -Z_{i j}^{3}\left(1+3 \frac{E_{i j}}{T_{i j}^{4}}\right)
\end{array}\right),
$$

3) In particular, we haven't changed the local error estimator. In this respect the current solver is still a prototype.

4) http://www.netlib.org/ode/vodpk.f 
where $b_{0}$ is a VODPK coefficient. Note that as in IMEX there is no grid connectivity used in this preconditioner. Default VODPK allows maximally five GMRES iterations per linear solve. It then requires 20 arrays of storage, including the workspace for the preconditioner.

\subsubsection{Summary of results}

Results are summarized in Figure 4.2 where we plot on a $\log$-log scale temporal $L_{2}$-errors (vertical axis) against measured CPU seconds (SUN Sparc Ultra4) for the chosen local error tolerances Tol. The horizontal dashed lines represent the spatial $L_{2}$-errors (in the left plot this line coincides with the upper border). Of course, it makes no sense to achieve temporal errors much smaller than their spatial counterparts. For $h=1 / 50,1 / 100,1 / 200$, the spatial $L_{2}$-errors are approximately $1.010^{-1}, 3.010^{-2}, 8.010^{-3}$, respectively. Hence the solvers should work reliably for coarse temporal tolerances. For presentation of the results five values for $T o l$ have been used. Both RKC and IMEX succeeded for $T o l=10^{-k}, k=$ $1(1 / 2) 3$. VODPK however failed for coarse tolerances, so that results for this solver were obtained with $T o l=10^{-k}, k=3(1 / 2) 5$. On the $200 \times 200$ grid VODPK also failed for $T o l=10^{-3}$. Unless noted otherwise, this solver was called with default user parameters.
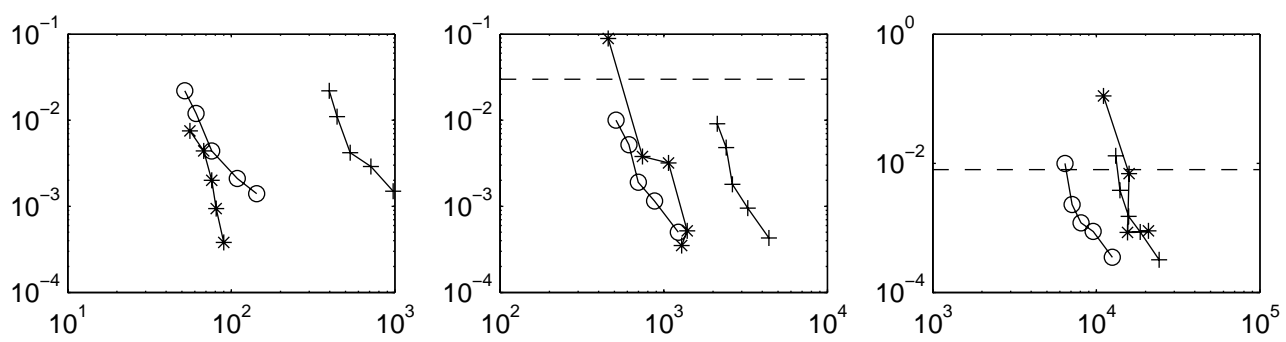

Figure 4.2: Temporal $L_{2}$-errors versus CPU seconds for $h=1 / 50$ (left), $h=1 / 100$ (middle) and $h=1 / 200$ (right). Solid lines -+- refer to RKC, solid lines -o- to IMEX and solid lines -*- to VODPK. The horizontal dashed lines give the spatial error level (for $h=1 / 50$ this line coincides with the upper border)

RKC versus IMEX. Comparing the two Runge-Kutta-Chebyshev solvers RKC and IMEX, we see that the latter is considerably faster as long as $\rho_{D} \ll \rho_{R}$, as anticipated. On the $50 \times 50$ grid the gain factor with respect to CPU time ranges from about 6 to 7 , on the $100 \times 100$ grid from about 3 to 4 , and on the $200 \times 200$ grid the gain factor is still about 2 . Of further interest is that the delivered accuracies and the required number of integration steps of the two solvers are very close (for a given $T o l$ and grid). The main difference is that the fully explicit solver requires much more stages per step for stability. We emphasize that on all three grids $T o l=10^{-1}$ is in fact small enough in view of the spatial error. Table 4.1 collects integration data for the IMEX solver.

IMEX versus VODPK. Comparing IMEX with VODPK, we conclude that the IMEX solver is much more reliable, is nearly equally efficient on the $50 \times 50$ grid (for the wanted coarse tolerances), more efficient on the $100 \times 100$ grid and substantially more efficient on the $200 \times 200$ grid. IMEX can be used with a very coarse tolerance which is desirable in view of the size of the spatial error. VODPK however readily fails for coarse tolerances and even for finer tolerances its accuracy behaviour is somewhat unpredictable. On the finest grid 


\begin{tabular}{l|lll}
\hline Tol & $h=1 / 50$ & $h=1 / 100$ & $h=1 / 200$ \\
\hline $10^{-1}$ & $1816(32+2,76)$ & $4601(54+2,115)$ & $12769(87+11,190)$ \\
$10^{-2}$ & $2589(67+2,80)$ & $6151(100+2,69)$ & $15925(169+2,112)$ \\
$10^{-3}$ & $4774(175+25,39)$ & $10840(258+26,49)$ & $24466(379+15,72)$ \\
\hline
\end{tabular}

Table 4.1: Integration data for the IMEX solver given as $N_{\text {stage }}\left(N_{a c c}+N_{r e j}, s_{\max }\right)$, where $N_{\text {stage }}$ is the total number of stages, $N_{a c c}$ the number of accepted steps, $N_{r e j}$ the number of rejected steps, and $s_{\max }$ the maximal number of stages per time step.

the explicit solver RKC is even competitive with VODPK. We also observed a substantial amount of linear convergence failures and therefore have increased the default maximum of 5 GMRES iterations to 10 . This indeed leads to a smoother process with substantially less linear convergence failures, but in general it did not result in a reduced CPU time. In some cases we observed some gain, but in other cases we lost CPU time (and detected even a total failure on the $100 \times 100$ grid for $T o l=10^{-3}$ ). For the present problem the default of 5 iterations is as good as 10 iterations and often even better. For an improved and (probably) more robust performance of VODPK it seems necessary to implement a substantially more complicated (and more expensive) preconditioner.

\section{Final remarks}

The original second-order solver RKC from [15] is fully explicit, stabilized, and requires little memory. This makes it an attractive, user-friendly tool for integrating large-scale semi-discrete parabolic problems. Its limitation lies in the stiffness and hence for efficiency reasons RKC should not be advocated for severely stiff semi-discrete parabolic problems. By treating reaction terms implicitly and diffusion terms still explicitly (IMEX approach), this limitation has been removed for severely stiff diffusion-reaction problems where the severe stiffness emanates from reaction terms having a Jacobian matrix with a real spectrum. By extending the solver with the IMEX option, it remains user-friendly and memory usage is still low. For the current radiation-diffusion problem the solver compares very favourably with VODPK, both with respect to robustness and efficiency.

\section{References}

[1] A. Abdulle, A.A. Medovikov (2001), Second order Chebyshev methods based on orthogonal polynomials. Numer. Math. 90, pp. 1-18.

[2] A. Abdulle (2002), Fourth order Chebyshev methods with recurrence relation. SIAM J. Sci. Comput. 23, pp. 2042-2055.

[3] M. Bakker (1971), Analytical aspects of a minimax problem (in Dutch). Technical Note TN 62, Mathematical Centre, Amsterdam.

[4] P.N. Brown, A.C. Hindmarsh (1989), Reduced storage matrix methods in Stiff ODE Systems, J. Appl. Math. Comput. 31, pp. 40-91. 
[5] P.N. Brown, C.S. Woodward (2001), Preconditioning strategies for fully implicit radiation diffusion with material-energy transfer, SIAM J. Sci. Comput. 23, pp. 499-516.

[6] G.D. Byrne (1992), Pragmatic experiments with Krylov methods in the stiff ODE setting, Computational Ordinary Differential Equations, J. Cash, I. Gladwell (eds.), Oxford Univ. Press, Oxford, pp. 323-356.

[7] P.J. van der Houwen, B.P. Sommeijer (1980), On the internal stability of explicit, $m$ stage Runge-Kutta methods for large m-values. Z. Angew. Math. Mech. 60, pp. 479485.

[8] W. Hundsdorfer, J.G. Verwer (2003), Numerical Solution of Time-Dependent Advection-Diffusion-Reaction Equations, Springer Series in Computational Mathematics, Vol. 33, Springer, Berlin (to appear in July).

[9] V.I. Lebedev (1994), How to solve stiff systems of differential equations by explicit methods. In: Numerical Methods and Applications. Ed. G.I. Marchuk, CRC Press, pp. $45-80$.

[10] V.I. Lebedev (2000), Explicit difference schemes for solving stiff problems with a complex or separable spectrum. Comput. Math. and Math. Phys. 40, pp. 1801-1812.

[11] V.A. Mousseau, D.A. Knoll, W.J. Rider (2000), Physics-based preconditioning and the Newton-Krylov method for non-equilibrium radiation diffusion. J. Comput. Phys. 160, pp. $743-765$.

[12] Y. Saad, M. Schultz (1986), GMRES: A generalized minimal residual algorithm for solving nonsymmetric linear systems. SIAM J. Sci. Statist. Comput. 7, pp. 856-869.

[13] L.F. Shampine (1994), Numerical Solution of Ordinary Differential Equations. Chapman \& Hall, New York.

[14] B.P. Sommeijer, J.G. Verwer, A performance evaluation of a class of Runge-KuttaChebyshev methods for solving semi-discrete parabolic differential equations. Report NW91/80, Mathematisch Centrum, Amsterdam (1980).

[15] B.P. Sommeijer, L.F. Shampine, J.G. Verwer (1997), RKC: An explicit solver for parabolic PDEs. J. Comput. Appl. Math. 88, pp. 315-326.

[16] J.G. Verwer, W.H. Hundsdorfer, B.P. Sommeijer (1990), Convergence properties of the Runge-Kutta-Chebyshev method. Numer. Math. 57, pp. 157-178. 